Received: 2016.12 .17

Accepted: 2016.02 .21

Published: 2017.10.20

Authors' Contribution:

A Study Design

B Data Collection

C Statistical Analysis

D Data Interpretation

E Manuscript Preparation

F Literature Search

G Funds Collection

\section{Magnetic Resonance (MR) Imaging Assessment for Glossopharyngeal Neuralgia: Value of Three-Dimensional T2-Reversed MR Imaging (3D-T2R) in Conjunction with Other Modes of 3D MR Imaging}

\author{
Masashi Nishihara ${ }^{1 \mathrm{BCDEF}}$, Tomoyuki Noguchi, ${ }^{1,2 \mathrm{AD}}$, Masatou Kawashima ${ }^{3 \mathrm{GE}}$, \\ Shinya Azama ${ }^{1 \mathrm{EG}}$, Ken Matsushima ${ }^{3 \mathrm{BDF}}$, Hiroyuki Irie ${ }^{1 \mathrm{AE}}$ \\ 1 Department of Radiology, Faculty of Medicine and Graduate School of Medicine, Saga University, Saga, Japan \\ 2 Department of Radiology, National Center for Global Health and Medicine, Tokyo, Japan \\ ${ }^{3}$ Department of Neurosurgery, Faculty of Medicine and Graduate School of Medicine, Saga University, Saga, Japan
}

Author's address: Tomoyuki Noguchi, Department of Radiology, Faculty of Medicine and Graduate School of Medicine, Saga University, 5-1-1, Nabeshima, Saga, 849-8501, Japan, e-mai: tnogucci@radiol.med.kyushu-u.ac.jp

Source of support: This work was partly supported by a Grant-in-Aid for Scientific Research from Japan Society for the Promotion of Science (№.26461856)

\section{Summary}

Background: $\quad$ To retrospectively examine the usefulness of gray-scale reversal imaging of T2-weighted images (3D-T2R) in conjunction with other modes of 3D MRI for preoperative assessments in patients with glossopharyngeal neuralgia (GPN) due to neurovascular compression.

Material/Methods: $\quad$ Imaging findings on 3D-T2R, constructive interference in steady state (CISS), and MRA were analyzed with reference to operative charts in 10 patients with GPN.

Results: $\quad$ Offending vessels were associated with the posterior inferior cerebellar artery (PICA) in 9 of 10 patients (90\%). Eight of the 10 patients $(80 \%)$ had offending vessels located at the supraolivary fossette. Of those eight patients, six (75\%) had a shift of the ipsilateral vertebral artery to the affected side. Five (42\%) and seven (48\%) contact points were associated with the root entry/exit zone and the peripheral nerve system segment, respectively. In six of nine contact points (67\%), 3D-T2R demonstrated the pathomorphological features at the contact points better than CISS.

Conclusions: The offending vessels were mostly associated with posterior inferior cerebellar arteries, were frequently located at the supraolivary fossette, and had attachments at the root entry/exit zone and at the peripheral segment of the glossopharyngeal nerve, which was well demonstrated on 3D-T2R.

MeSH Keywords: $\quad$ Decompression • Glossopharyngeal Nerve Diseases • Magnetic Resonance Imaging

PDF file: $\quad$ http://www.polradiol.com/abstract/index/idArt/902896

\title{
Background
}

Glossopharyngeal neuralgia (GPN) is a rare disease that causes severe episodic pain, lasting from several seconds to several minutes, in the tonsils, larynx, tongue, and/or ears that are innervated by somatosensory elements of the glossopharyngeal and vagus nerves [1]. GPN can be induced by several factors such as tumor [2], infarction [3], Chiari I malformation [4], or dissection of the vertebral artery [5].
Neurovascular compression is also one of the important causes of GPN [6]. Medications such as carbamazepine remain the first choice of treatment for patients with GPN due to neurovascular compression. Microneurovascular decompression surgery is performed for GPN in patients who have an unsatisfactory response to medications or suffer from side effects of the drugs $[7,8]$. 
Table 1. Characteristics of patients.

\begin{tabular}{cccl}
\hline Patient No. & Age (year)/sex & Side & \multicolumn{1}{c}{ Symptom } \\
\hline 1 & $41 /$ Male & Left & Pharyngeal pain, ear pain \\
\hline 2 & $48 /$ Male & Left & Pharyngeal pain, ear pain \\
\hline 3 & $56 /$ Male & Right & Pharyngeal pain, tongue pain \\
\hline 4 & $62 /$ Male & Left & Pharyngeal pain \\
\hline 5 & $53 /$ Female & Left & Pharyngeal pain \\
\hline 6 & $58 /$ Female & Left & Pharyngeal pain, ear pain, maxillary pain \\
\hline 7 & $64 /$ Female & Left & Pharyngeal pain \\
\hline 8 & $66 /$ Female & Right & Pharyngeal pain, ear pain \\
\hline 9 & $67 /$ Female & Left & Pharyngeal pain, ear pain \\
\hline 10 & $69 /$ Female & Right & Pharyngeal pain \\
\hline
\end{tabular}

Magnetic resonance imaging (MRI) is very useful for initial screening in GPN cases and for preoperative imaging assessment to identify offending vessels, their course, and contact points and to characterize the condition of the glossopharyngeal nerve. MR images with three-dimensional data acquisition (3D-MRI) are recommended, and they include 3D time-of-flight magnetic resonance angiography (MRA) and constructive interference in steady state (CISS) [9-12].

Fujii et al. [13] proposed that high resolution T2-weighted images obtained on a 3.0-T MRI unit can provide improved structural and contrast resolution by processing for optimum perceptual resolution through the application of gray-scale reversal and an expansion of the gray-scale window, known as reversed (T2R) imaging. We applied the T2R imaging technique to 3D-T2-weighted images by using the 3D sampling perfection along with the application of optimized contrasts using a different flip angle evolution sequence (3D-T2R). The results demonstrated that 3D-T2R was useful for detecting various physiological and pathological conditions that give rise to GPN. In the present study, we retrospectively examined 3D-T2R images in conjunction with other modes of 3D MRI at 3.0-T for preoperative evaluation of GPN due to neurovascular compression.

\section{Material and Methods}

The current retrospective study was approved by our institutional review board (No.2013-11-07), and written informed consent from all patients was waived.

\section{Patients}

Between February 2007 and September 2013, we enrolled 10 patients with typical GPN due to neurovascular compression who achieved symptom relief after microvascular decompression surgery (Table 1). There were four males and six females; their age range and mean age were 48-69 and 58.4 years, respectively. The right side was affected in three patients, and the left side was affected in the other seven patients.

\section{Imaging conditions}

3D-T2R, CISS, and MRA were performed preoperatively in all patients on a clinical 3.0-Tesla MRI unit (MAGNETOM Trio, A Tim System, Siemens AG, Erlangen, Germany) with a 12-channel head coil. The range and median of the time interval between the MRI examination and the microvascular decompression surgery were 6-94 days and 7 days, respectively. The imaging parameters were as follows:

1) 3D-T2R: TR/TE $(\mathrm{ms})=1300 / 106$; flip angle (degree) $=120$; matrix size $=640 \times 640$; slice thickness $(\mathrm{mm})=0.6$; scan height $(\mathrm{mm})=40$ to 60 (the region covering medulla, pons, and midbrain); number of partitions $=67$ to 100 .

2) CISS: Repetition time (TR)/Echo time (TE) $(\mathrm{ms})=5.80$ to $6.99 / 2.5$ to 3.0 ; flip angle (degree) $=48$; matrix size $=320 \times 320$ to $512 \times 512$; slice thickness $(\mathrm{mm})=0.5$ to 0.8 ; scan height $(\mathrm{mm})=40$ to 60 (the region covering medulla, pons, and midbrain); number of partitions $=40$ to 120 .

3) MRA: TR/TE $(\mathrm{ms})=22 / 3.1$; flip angle (degree) $=18$; matrix size $=384 \times 320$; slice thickness $(\mathrm{mm})=0.7$ to 0.8 ; scan height $(\mathrm{mm})=71.4$ to $96 \mathrm{~mm}$ (the region covering from the foramen magnum to the circle of Willis); number of partitions $=102$ to 120 .

Reconstructed coronal and sagittal oblique images from both 3D-T2R and CISS, and maximum intensity projection (MIP) images from MRA were also generated and used for the imaging evaluation.

\section{Imaging evaluations}

The following imaging findings on 3D-T2R, CISS, and MRA were analyzed by two radiologists (TN with 11 years of experience in neuroradiology; MN with 7 years of experience in neuroradiology) in consensus with reference to the operative charts.

\section{Identification of offending vessels}

The offending vessels were investigated by identifying both the contact(s) with the glossopharyngeal nerve on 3D-T2R and CISS and the anatomical origin on MRA. The offending vessels and the associated pathological states, such as 


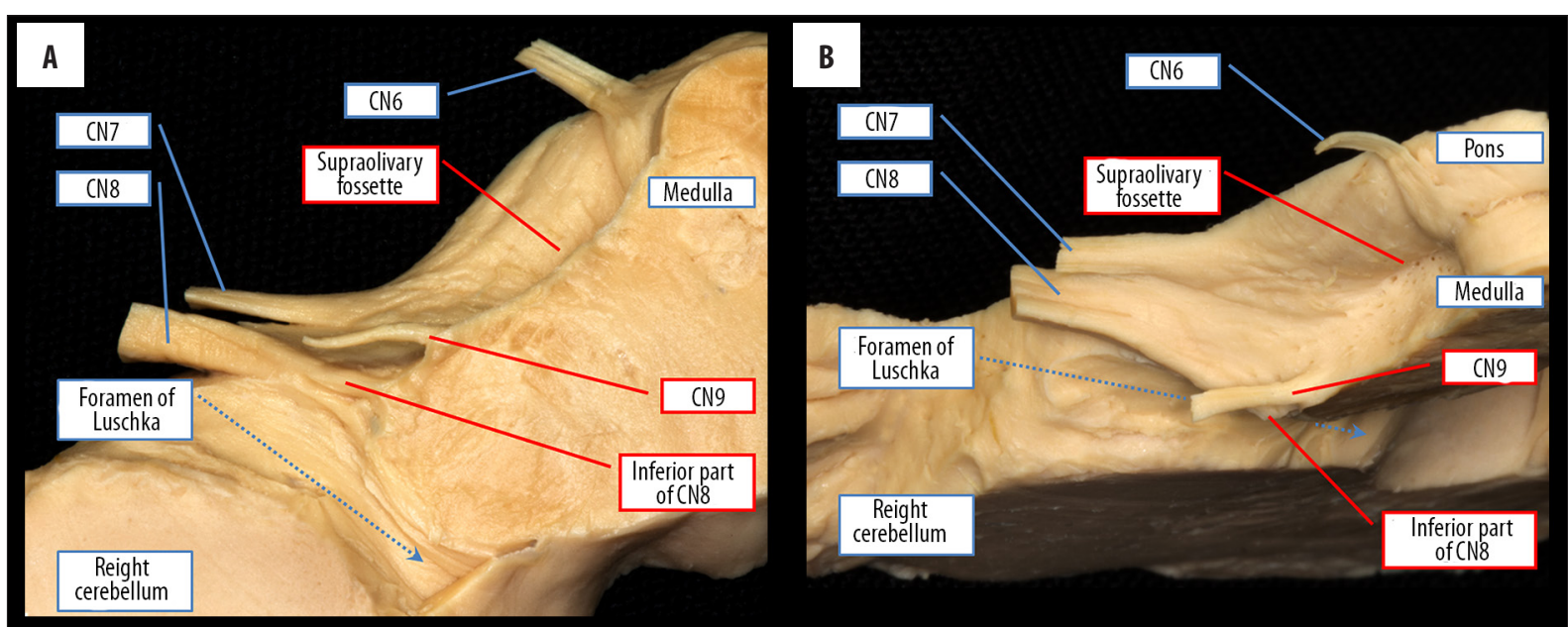

Figure 1. The bottom-to-top-view (A) and the anterior-to-posterior-view (B) of a normal cadaver specimen at the level of the ponto-medullary junction. The right glossopharyngeal nerve (CN9) is detected as a single, thin nerve root ahead of the inferior part of the cochlear nerve (CN8) root originating from the brain stem. The supraolivary fossette is located in the bulbopontine sulcus between the abducens nerve (CN6) and the facial nerve (CN7).

the presence of the adhesive arachnoiditis, were validated based on the operative charts.

\section{The courses of the offending vessels and the ipsilateral vertebral artery}

We investigated whether the offending vessels coursed through the supraolivary fossette because previous reports revealed that finding in patients with GPN due to neurovascular compression $[8,10]$. We also investigated the presence of a shift of the ipsilateral vertebral artery to the affected side, which might indirectly lead to neurovascular compression by rendering cerebellar arteries tortuous.

\section{Identification of contact point}

First, the glossopharyngeal nerve was identified on CISS and 3D-T2R according to the combination of three methods, as follows:

1) Identification of the proximal side of the glossopharyngeal nerve: Ahead of the inferior part of the cochlear nerve root, a single nerve root of the glossopharyngeal nerve originates from the brain stem (Figure 1);

2) Identification of the morphology of the glossopharyngeal nerve: The glossopharyngeal nerve, i.e., a single fiber of the glossopharyngeal nerve with a diameter of $<1 \mathrm{~mm}$ [14] (Figure 2);

3) Identification of the distal side of glossopharyngeal nerve: A single fiber of the glossopharyngeal nerve is located in the anterior part of the jugular foramen [15].

The attachments of the glossopharyngeal nerve with offending vessels were then identified on 3D-T2R and CISS with reference to the operative charts.

Finally, the distance between the glossopharyngeal nerve origin and the contact point was measured and divided into two parts based on the following criteria proposed by De Ridder et al. [16].
A) Root entry/exit zone (REZ): < $1.1 \mathrm{~mm}$ distance between the contact point and nerve origin;

B) Peripheral nerve system segment (PNS): $\geq 1.1 \mathrm{~mm}$ distance between the contact point and the nerve origin.

\section{Shift of the glossopharyngeal nerve at the contact point(s)}

We determined the presence/absence of a shift of the glossopharyngeal nerve at the contact point which would suggest that neurovascular compression caused GPN.

\section{Comparison between the diagnostic capabilities of CISS and 3D-T2R}

We compared the capabilities of 3D-T2R and CISS for identifying the glossopharyngeal nerve, the offending vessel at the contact point, and the glossopharyngeal nerve within the cistern.

\section{Results}

The results are summarized in Table 2.

\section{Identification of offending vessels}

The offending vessels that had been recorded in the operative charts were mostly associated with the posterior inferior cerebellar artery (PICA), i.e., in nine of the 10 patients (90\%); PICA was the only affected vessel in seven patients (70\%); a combination of PICA and the vertebral artery (VA) was observed in one patient (10\%), and a combination of PICA and the anterior inferior cerebellar artery (AICA) was observed in one (10\%) patient. The offending vessel in the remaining patient (10\%) was AICA alone. Consistency in the identification of offending vessels by 3D-T2R, CISS, MRA, and operative charts was observed in only seven patients (except patients no. 2,6, and 7).

\section{Courses of the offending vessels and the ipsilateral vertebral artery}

In eight patients $(80 \%)$, the offending vessels were located at the supraolivary fossette, which was observed on 


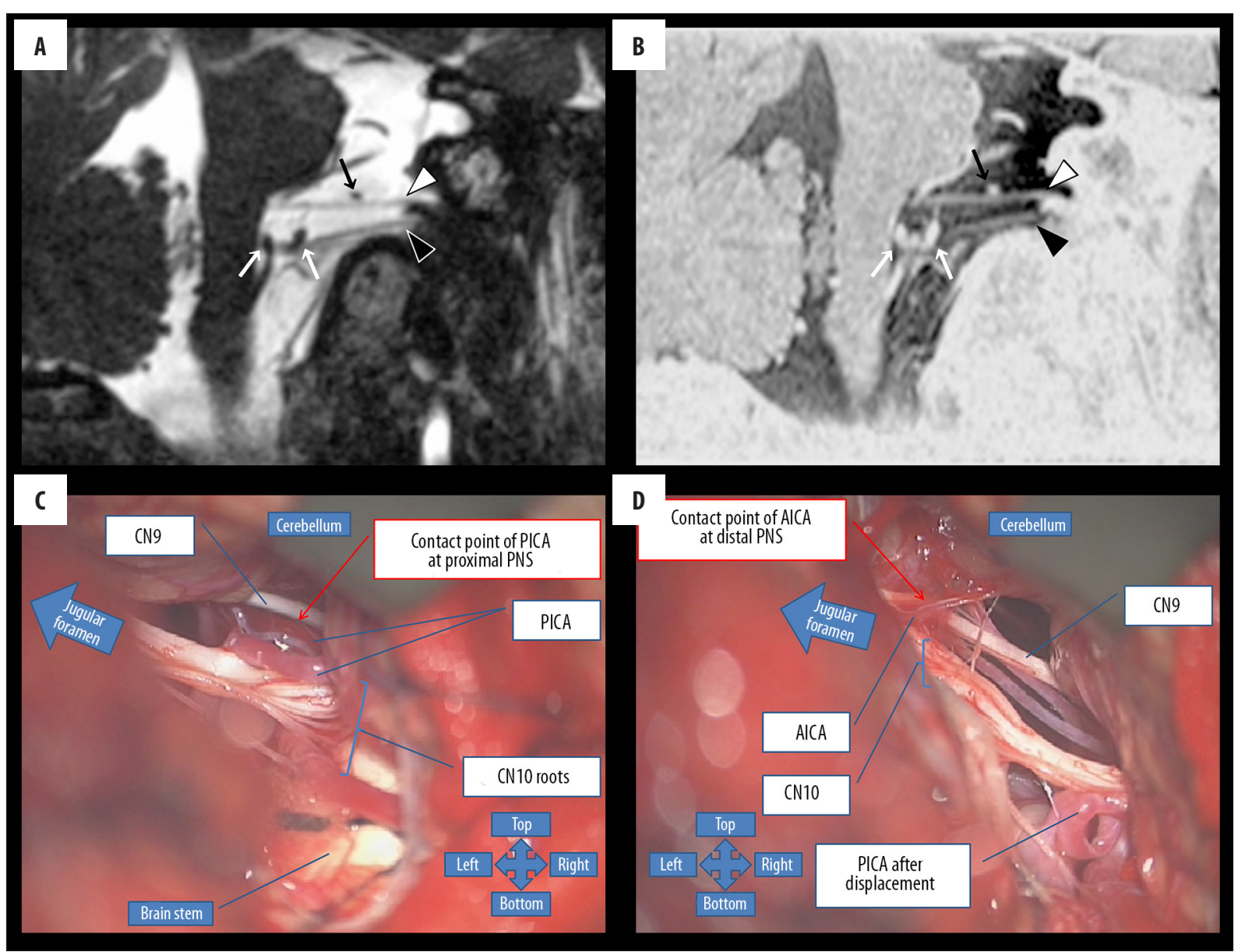

Figure 2. A 48-year-old man (no. 2) with left glossopharyngeal neuralgia. Sagittal-oblique CISS (A) and 3D-T2R images (B) demonstrate that a single thin fiber of the glossopharyngeal nerve $(\mathbf{A}, \mathbf{B}$; white arrowhead) located between the brainstem and the anterior part of the jugular foramen attaches to AICA (A, B; black arrow) without a nerve shift at distal PNS, but it does not contact PICA (A, B; white arrow) at proximal PNS. Intraoperative photographs (C, D) show that PICA and AICA had attachments with the glossopharyngeal nerve at the proximal and distal PNS. In addition, sagittal-oblique CISS and 3D-T2R images clearly display the vagus nerve (A, B; black arrowhead) between the brainstem and the posterior part of the jugular foramen.

3D-T2R and CISS. Of those eight patients, six $(75 \%)$ had a shift of the ipsilateral vertebral artery to the affected side, as seen on 3D-T2R and CISS. The other 2 patients (no. 6 and 10) did not have the offending vessels in the supraolivary fossette or a shift of the ipsilateral vertebral artery to the affected side.

\section{Contact points}

Of the 12 contact points that were observed intraoperatively, five $(42 \%)$ were associated with REZ, and seven (58\%) were associated with PNS; REZ alone in four patients $(40 \%)$, PNS alone in four patients (40\%); both REZ and PNS in one patient $(10 \%)$; the proximal and distal parts of PNS in one patient (10\%). Nine of the 12 contact points identified intraoperatively (75\%) were also observed on both CISS and 3D-T2R. However, the other three contact points of three patients were not found. In one patient (no. 2) with PICA and AICA attached to the proximal and distal parts of PNS, only the proximal attachment was observed on CISS and 3D-T2R (Figure 2). Another patient (no. 6) with a contact point at PNS in conjunction with adhesive arachnoiditis, had no contact points between PICA or any other vessels and the glossopharyngeal nerve on MRI. Additionally, he had no remarkable imaging findings of adhesive arachnoiditis on MRA, CISS, or 3D-T2R. Another patient (no. 7) had no contact point with PICA at REZ of the glossopharyngeal nerve traversing through the cerebellomedullary cistern, but it touched the cerebellum, as seen on CISS and 3D-T2R.

\section{Shift of the glossopharyngeal nerve at the contact points}

Of the eight patients with offending vessels attaching to the glossopharyngeal nerve at contact points observed on both CISS and 3D-T2R, seven had a shifted glossopharyngeal nerve at the contact points: posteriorly in three patients; superiorly in one; inferiorly in one; anteroinferiorly in one; posterosuperiorly in one.

\section{Comparison between diagnostic capabilities of CISS and 3D-T2R}

The glossopharyngeal nerves in the cistern were clearly visualized in all 10 patients on both CISS and 3D-T2R. 
Table 2. Intraoperative and MRI findings.

\begin{tabular}{|c|c|c|c|c|c|c|c|c|c|}
\hline \multirow[b]{2}{*}{$\begin{array}{l}\text { Patient } \\
\text { No. }\end{array}$} & \multicolumn{3}{|c|}{ Intraoperative findings } & \multicolumn{3}{|c|}{ MRI findings } & \multirow[b]{2}{*}{$\begin{array}{l}\text { Correspondence } \\
\text { between } \\
\text { intraoperative } \\
\text { and MRI findings }\end{array}$} & \multicolumn{2}{|c|}{ Diagnostic capability } \\
\hline & $\begin{array}{l}\text { Offending } \\
\text { vessel }\end{array}$ & $\begin{array}{l}\text { Contact } \\
\text { point }\end{array}$ & $\begin{array}{l}\text { Additional } \\
\text { findings }\end{array}$ & $\begin{array}{l}\text { Offending } \\
\text { vessel } \\
\text { through SOF }\end{array}$ & $\begin{array}{c}\text { Shift of } \\
\text { ipsilateral } \\
\text { VA } \\
\text { to affected } \\
\text { side }\end{array}$ & $\begin{array}{l}\text { Shift of } \\
\text { CN9 at } \\
\text { contact } \\
\text { point }\end{array}$ & & $\begin{array}{c}\text { CN9 } \\
\text { in cistern }\end{array}$ & $\begin{array}{l}\text { CN9 and } \\
\text { offending } \\
\text { vessel } \\
\text { at contact } \\
\text { point }\end{array}$ \\
\hline 1 & PICA & PNS & - & Yes & Yes & Yes & Yes & Equal & $3 D-T 2 R$ \\
\hline 2 & $\begin{array}{l}\text { PICA and } \\
\text { AICA }\end{array}$ & $\begin{array}{l}\text { Proximal and } \\
\text { distal PNS }\end{array}$ & - & Yes & Yes & No & $\begin{array}{l}\text { No } \\
\text { Yes }\end{array}$ & Equal & Equal \\
\hline 3 & AICA & PNS & - & Yes & Yes & Yes & Yes & CISS & 3D-T2R \\
\hline 4 & $\begin{array}{l}\text { PICA and } \\
\text { VA }\end{array}$ & PNS & - & Yes & Yes & Yes & Yes & Equal & $3 \mathrm{D}-\mathrm{T} 2 \mathrm{R}$ \\
\hline 5 & PICA & REZ & - & Yes & Yes & Yes & Yes & Equal & Equal \\
\hline 6 & PICA & PNS & $\begin{array}{l}\text { Adhesive } \\
\text { arachnoiditis }\end{array}$ & No & No & N.a. & No & Equal & N.A. \\
\hline 7 & PICA & REZ & - & Yes & No & N.a. & No & CISS & N.A. \\
\hline 8 & PICA & REZ & - & Yes & Yes & Yes & Yes & CISS & Equal \\
\hline 9 & PICA & $\begin{array}{l}\text { REZ and } \\
\text { PNS }\end{array}$ & - & Yes & No & Yes & $\begin{array}{l}\text { Yes } \\
\text { Yes }\end{array}$ & Equal & 3D-T2R \\
\hline 10 & PICA & REZ & - & No & No & Yes & Yes & Equal & 3D-T2R \\
\hline
\end{tabular}

AICA - anterior inferior cerebellar artery; CN9 - glossopharyngeal nerve; N.A. - not applicable; PICA - posterior inferior cerebellar artery; PNS - peripheral nerve system segment; REZ - root entry/exit zone; SOF - supraolivary fossette; VA - vertebral artery.

With respect to six of nine contact points (67\%) in eight patients, 3D-T2R provided more distinct contrast between the glossopharyngeal nerves and the offending vessels than CISS. The other three contact points (33\%) were seen equally well on 3D-T2R and CISS. Additionally, in three patients $(30 \%)$, CISS was found to provide a better demonstration of the glossopharyngeal nerve in the cistern than 3D-T2R because of sharper nerve margin delineation. The other seven cases (70\%) were judged to be equally well visible on CISS and 3D-T2R.

Figure 2 (patient no. 8) and Figure 3 (patient no. 2) illustrate representative cases.

\section{Discussion}

MRI is very useful for investigating anatomical details in the posterior cranial fossa [9]. Previous studies recommended 3D time-of-flight MR angiography (3D-TOF-MRA), CISS [9-12], high-resolution two-dimensional T2-weighted imaging [1 1,17], 3D fast spin echo T2- weighted imaging,[9] 3D fast imaging with steady-state free precession MR angiography (3D-FISP-MRA) [11], high-resolution short tau inversion recovery (STIR) [18], and gadolinium-enhanced 3D spoiled gradient recalled acquisition at the steady state (SPGR) $[9,19]$. In particular, CISS was reported to be useful in detecting nerves and other structures [9-12], as CISS produces strongly T2-weighted images. Unlike other gradient echo sequences, such as gradient recalled acquisition in steady state (GRASS) or fast imaging with steady state precession (FISP), CISS has inherent flow compensation, which greatly reduces cerebrospinal fluid (CSF) pulsation artifacts that could obscure cisternal details [20]. In fact, in three of the 10 patients in the present study, CISS provided more sharper delineation of the margin of the glossopharyngeal nerve in the cistern compared to 3D-T2R. In contrast, 3D-T2R was better than CISS at demonstrating the contrast of the glossopharyngeal nerve and the offending vessel at six of nine contact points in eight patients. Unlike CISS, 3D-T2R provides low signal intensity for liquids such as cerebrospinal fluid, high signal intensity for flow voids such as blood vessels, and intermediate signal intensity for brain parenchyma or cranial nerves, which can create good contrast at the boundaries between blood vessels and nerves. Therefore, it was more suitable to evaluate the details around the contact points with 3D-T2R than CISS.

De Ridder et al. speculated that REZ of the glossopharyngeal nerve can be the contact point causing GPN due to neurovascular compression [16]. Their hypothesis is based on the histological characteristics of cranial nerves. Axons of the cranial nerves are covered by a myelin sheath composed of two histological cell types, oligodendrocytes and Schwann cells. Oligodendrocytes are found predominantly in the central zone, i.e., REZ, and Schwann cells are found in the peripheral zone, i.e., PNS. Oligodendrocytes have comparatively sparse connective tissue, while Schwann cells have relatively dense connective tissue. For this reason, the nerve compression in REZ can produce the symptoms of GPN. 


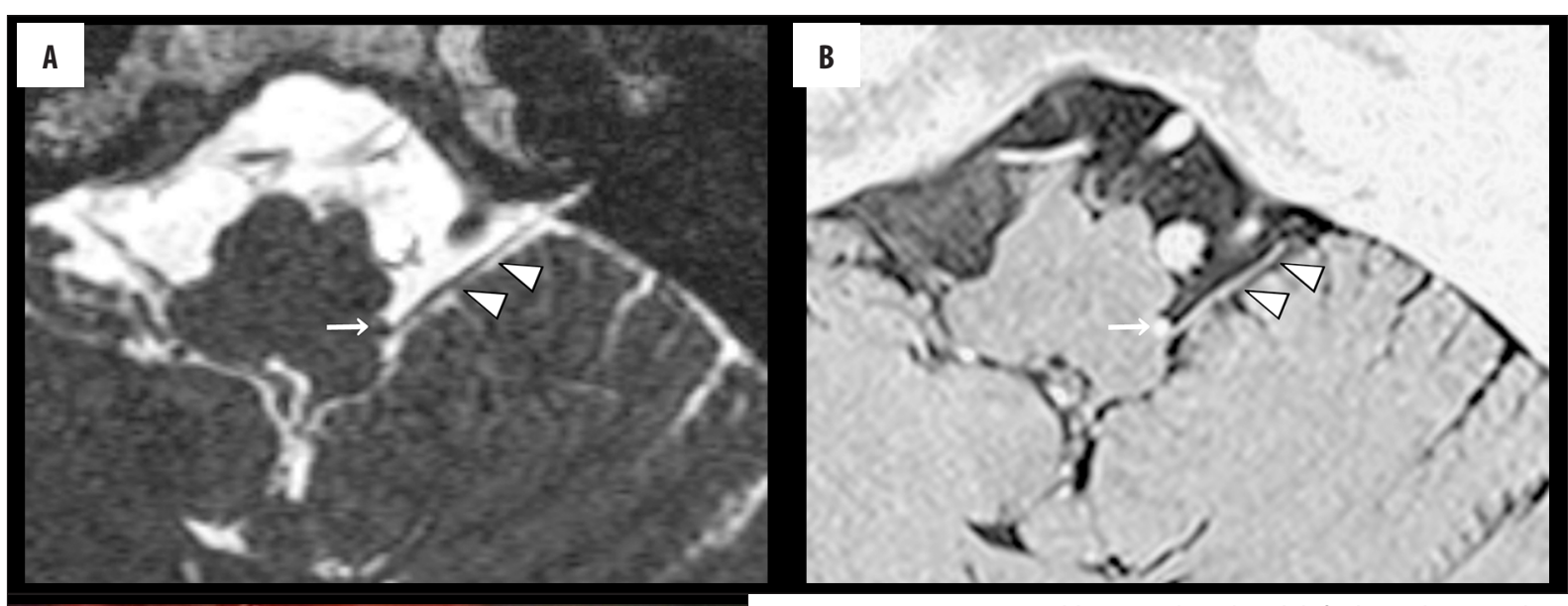

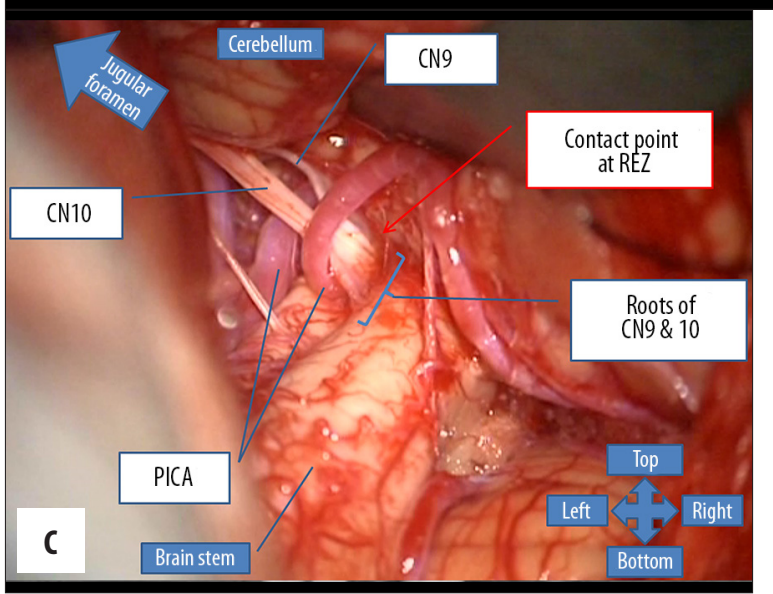

On the other hand, Boch et al. reported that compression can occur both in the PNS segment and REZ [9]. In our study, five contact points at REZ and seven contact points at PNS were detected intraoperatively, suggesting that GPN might occur even due to compression of the not-fragile zone. Our analysis also showed that seven of eight cases (88\%) had a shift of the glossopharyngeal nerve at the contact point. These results suggest the following two possibilities: (1) the distribution of Schwann cells and oligodendrocytes varies among individuals, and the length of the fragile zone consisting of oligodendrocytes might be $>1.1 \mathrm{~mm}$; (2) the peripheral compression indirectly pulls the fragile zone along the nerve root. Further assessments are needed to test these hypotheses.

In previous reports in patients with GPN, the offending vessels were thought to be PICA, AICA, VA, veins, or combinations of those vessels $[6,8,10,12,21]$. PICA is the most frequently reported offending vessel and has been described as running through the supraolivary fossette $[8,10]$. Our findings are consistent with these previous reports. In addition, we found that six of the eight patients with offending vessels coursing through the supraolivary fossette had a shift of the ipsilateral vertebral artery to the affected side, which could provoke glossopharyngeal neuralgia due to nerve compression.

This study has several limitations. It was retrospective and enrolled a small number of patients. There is a possibility of observer bias in the assessment of MR images due to lack of blinding. Therefore, further prospective
Figure 3. A 53-year-old woman (no. 5) with left glossopharyngeal neuralgia. Axial CISS (A) and 3D-T2R images (B) show that the left glossopharyngeal nerve (A, $\mathbf{B}$; white arrowheads) traverses between the brainstem and the anterior part of the jugular foramen in contact with PICA (A, B; white arrow) at REZ, with a nerve shift in the posterior direction. An intraoperative photograph (C) demonstrates a tortuous PICA displacing the roots of the glossopharyngeal and vagus nerves posteriorly at REZ.

investigations with larger samples should be performed to substantiate the results of our study. Only findings obtained by MRI and MRA without contrast material were investigated herein and therefore we could not evaluate the potential effects of veins as offending vessels $[6,8,10,12,21]$. Another limitation of our study is the discrepancy between MRI findings and operative charts. Intraoperative findings could be modified by the deformation of the parenchyma or the shifts of the vessels or cranial nerves caused by surgical manipulations such as brain retraction or cerebrospinal fluid suction. In fact, no attachments between the offending vessels and the glossopharyngeal nerve were observed on CISS or 3D-T2R in two of 12 contact points. In the future, endoscopic procedures might widen the operative view and help overcome this problem.

\section{Conclusions}

In patients with GPN, the offending arteries were mostly associated with PICA. Our series revealed that the offending vessels can come in contact with REZ and PNS of the glossopharyngeal nerve. Findings such as the cerebellar vessels coursing through the supraolivary fossette or a shift of the ipsilateral vertebral artery to the affected side might indicate neurovascular compression. 3D-T2R is useful for the preoperative assessment of contact points of the glossopharyngeal nerves with offending vessels in patients with GPN who need surgery, whereas CISS is helpful for detecting the glossopharyngeal nerve in the cistern.

\section{Conflicts of interest}

We wish to confirm that there are no known conflicts of interest associated with this publication and that there has been no significant financial support for this work that could have influenced its outcome. 


\section{References:}

1. Matsushima T, Goto Y, Ishioka $\mathrm{H}$ et al: Possible role of an endovascular provocative test in the diagnosis of glossopharyngeal neuralgia as a vascular compression syndrome. Acta Neurochir (Wien), 1999; 141: 1229-32

2. Phuong HL, Matsushima T, Hisada K, Matsumoto K: Glossopharyngeal neuralgia due to an epidermoid tumour in the cerebellopontine angle. J Clin Neurosci, 2004; 11: 758-60

3. Warren HG, Kotsenas AL, Czervionke LF: Trigeminal and concurrent glossopharyngeal neuralgia secondary to lateral medullary infarction. Am J Neuroradiol, 2006; 27: 705-7

4. Kanpolat Y, Unlu A, Savas A, Tan F: Chiari Type I malformation presenting as glossopharyngeal neuralgia: case report. Neurosurgery, 2001; 48: 226-28

5. Soga Y, Ito Y: Sudden onset pharyngeal pain associated with dissecting vertebral artery aneurysm. Acta Neurochir (Wien), 2002; 144: 835-38; discussion 838

6. Wakiya K, Fukushima T, Miyazaki S: [Results of microvascular decompression in 16 cases of glossopharyngeal neuralgia]. Neurol Med Chir (Tokyo), 1989 29: 1113-18 [in Japanese]

7. Matsushima T, Goto Y, Natori Y et al: Surgical treatment of glossopharyngeal neuralgia as vascular compression syndrome via transcondylar fossa (supracondylar transjugular tubercle) approach. Acta Neurochir (Wien), 2000; 142: 1359-63

8. Kawashima M, Matsushima T, Inoue T et al: Microvascular decompression for glossopharyngeal neuralgia through the transcondylar fossa (supracondylar transjugular tubercle) approach. Neurosurgery, 2010; 66: 275-80; discussion 280

9. Boch AL, Oppenheim C, Biondi A et al: Glossopharyngeal neuralgia associated with a vascular loop demonstrated by magnetic resonance imaging. Acta Neurochir (Wien), 1998; 140: 813-18

10. Hiwatashi A, Matsushima T, Yoshiura T et al: MRI of glossopharyngeal neuralgia caused by neurovascular compression. Am J Roentgenol, 2008; 191: 578-81

11. Schmitz SA, Hohenbleicher H, Koennecke HC et al: Neurogenic hypertension. A new MRI protocol for the evaluation of neurovascular compression of the cranial nerves IX and X root-entry zone. Invest Radiol, 1999; 34: 774-80
12. Karibe H, Shirane R, Yoshimoto T: Preoperative visualization of microvascular compression of cranial nerve IX using constructive interference in steady state magnetic resonance imaging in glossopharyngeal neuralgia. J Clin Neurosci, 2004; 11: 679-81

13. Fujii Y, Nakayama N, Nakada T: High-resolution T2-reversed magnetic resonance imaging on a high magnetic field system. Technical note. J Neurosurg, 1998; 89: 492-95

14. Guclu B, Sindou M, Meyronet D et al: Cranial nerve vascular compression syndromes of the trigeminal, facial and vagoglossopharyngeal nerves: Comparative anatomical study of the central myelin portion and transitional zone; correlations with incidences of corresponding hyperactive dysfunctional syndromes. Acta Neurochir (Wien), 2011; 153: 2365-75

15. Moon WJ, Roh HG, Chung EC: Detailed MR imaging anatomy of the cisternal segments of the glossopharyngeal, vagus, and spinal accessory nerves in the posterior fossa: the use of 3D balanced fastfield echo MR imaging. Am J Neuroradiol, 2009 30: 1116-20

16. De Ridder D, Moller A, Verlooy J et al: Is the root entry/exit zone important in microvascular compression syndromes? Neurosurgery, 2002; 51: 427-33; discussion 433-24

17. Fischbach F, Lehmann TN, Ricke J, Bruhn H: Vascular compression in glossopharyngeal neuralgia: demonstration by high-resolution MRI at 3 tesla. Neuroradiology, 2003; 45: 810-11

18. Hiwatashi A, Yoshiura T, Yamashita K et al: High-resolution STIR for 3-T MRI of the posterior fossa: visualization of the lower cranial nerves and arteriovenous structures related to neurovascular compression. Am J Roentgenol, 2012; 199: 644-48

19. Anderson VC, Berryhill PC, Sandquist MA et al: High-resolution three-dimensional magnetic resonance angiography and threedimensional spoiled gradient-recalled imaging in the evaluation of neurovascular compression in patients with trigeminal neuralgia: A double-blind pilot study. Neurosurgery, 2006; 58: 666-73; discussion 666-73

20. Laine FJ, Underhill T: Imaging of the lower cranial nerves. Magn Reson Imaging Clin N Am, 2002; 10: 433-49

21. Sampson JH, Grossi PM, Asaoka K, Fukushima T: Microvascular decompression for glossopharyngeal neuralgia: long-term effectiveness and complication avoidance. Neurosurgery, 2004; 54: 884-89: discussion 889-90 